\title{
RADIATION FROM A UNIFORMLY ACCELERATED CHARGE
}

\author{
Amos Harpaz ${ }^{1}$ \& Noam Soker ${ }^{1}$ \\ 1. Department of Physics, University of Haifa at Oranim, Tivon 36006, ISRAEL \\ phr89ah@vmsa.technion.ac.il \\ soker@physics.technion.ac.il
}

\begin{abstract}
The emission of radiation by a uniformly accelerated charge is analyzed. According to the standard approach, a radiation is observed whenever there is a relative acceleraion between the charge and the observer. Analyzing difficulties that arose in the standard approach, we propose that a radaition is created whenever a relative acceleration between the charge and its own electric field exists. The electric field induced by a charge accelerated by an external (nongravitational) force, is not accelerated with the charge. Hence the electric field is curved in the instantanous rest frame of the accelerated charge. This curvature gives rise to a stress force, and the work done to overcome the stress force is the source of the energy carried by the radiation. In this way, the "energy balance paradox" finds its solution.
\end{abstract}

key words: Principle of Equivalence, Curved Electric Field 


\section{Introduction}

The emission of radiation from a uniformly accelerated charge is considered to be a well solved problem. However, when the solution of this problem is treated in its relevance to the principle of equivalence, and to observations made by observers located in different frames of reference, some contradictions appear to exist in the solution.

In the standard spproach to the solution of this problem, which is mostly accepted in the physics community, radiation from a charged particle can be observed whenever there is a relative acceleration between the observer and the charge Rohrlich $^{(1)}$ 1965, see also Rohrlich ${ }^{(2)} 1963$, Coleman $^{(3)}$ 1961, Boulware ${ }^{(4)}$ 1980). This topic is relevant to the principle of equivalence in general relativity, which states the equivalence of a uniformly accelerated system of reference in

free space to an unaccelerated system, subjected to the action of a static homogenous gravitational field (SHGF). According to this principle, no physical observation can lead an observer to conclude whether he is located in a uniformly accelerated system in a gravity free space, or if he is at rest in a SHGF. This principle is used to analyze the possible observations in different situations, that enable to determine whether a radiation can be observed. Following the analysis of Rohrlich ${ }^{(1)}$ (1965), which presents the standard approach in a wide presentation, let us consider a charged particle falling freely in SHGF. According to the principle of equivalence, this particle is in an inertial system. In order to calculate the radiation from this particle, one should determine the electromagnetic fields of the charged particle.

According to Rohrlich ${ }^{(1)}$ (1965), there are three criteria for the existence of a radiation field:

(1) The source of the electric field is accelerating.

In a case that we only know the velocities and not the acceleration, we can use a second criterion:

(2) The field falls with the distance as $1 / r$.

However, the use of this criterion is convenient in the wave zone, where the distance from the 
source is larger than the wave length $(r \gg \lambda)$.

There is still another local criterion.

(3) For this criterion, one integrates the electromagnetic energy tensor over a sphere of radius $r$ that circumference the source, at time $t=t_{0}+r / c$, where the source was located at the center of the sphere at $t=t_{0}$. The vanishing of this integral shows that no radiation is emitted. The importance of this criterion is that it can be performed at any (short) distance from the source.

In $\S 2$ we analyze the standard approach and point to several weak points in this approach. In $\S 3$ we present an alternative approach, according to which radiation is created due to stress force that exists in the electric field of the accelerated charge, and in $\S 4$ the energy carried by the radiation is calculated, using the work done to overcome the stress force of the field.

\section{Analysis of the Standard Approach}

Using Maxwell's equations, we can calculate electromagnetic fields in inertial frames of reference (in flat space-time, using the tools of the special theory of relativity). These fields are then transformed to accelerated frames of reference (or to curved space-time by using the transformations of the general theory), and by these transformations the fields in the non-inertial systems can be found. In order to analyze the standard approach, consider an observer falling freely in an SHGF. A freely falling system of reference is an inertial system of reference for him. A charged particle falling freely parallel to the observer in the same SHGF is static relative to the freely falling observer. The observer will observe a static unperturbed culoumb field, and no radiation from the freely falling charge. A neutral particle, having an equal mass, falling freely in parallel to the charged particle, will fall exactly with the same acceleration and velocity as the charged particle, because both particles lose no energy by radiation, and the work done on the particles by the gravitational field that creates the kinetic energy of the particles is equal for both of them. The observer finds no difference between the response of the two particles to the SHGF. This observation supports the principle of equivalence, because the observer cannot distinguish between the situation in which he and the particles are falling freely in SHGF, or if all of them are 
located in a static gravity-free system of reference $\left(\right.$ Rohrlich $\left.^{(1)} 1965\right)$.

An observer located in a static laboratory in the gravitational field, will observe that the two particles are uniformly accelerated in the SHGF. In order to find the electromagnetic fields in his lab, he performs a transformation of the fields of the charged particle calculated in the inertial frame, to his system of reference. This transformation creates a magnetic field in the lab system, in addition to the change in the electric field caused by the transformation. According to the standard approach, the existence of these fields in the lab system gives rise to a radiation field, and the observer in the lab will observe this radiation, and conclude that a freely falling charge in SHGF radiates Rohrlich $^{(1)}$ 1965). Certainly, the neutral particle falling parallel to the charged particle emits no radiation.

However, this picture of the standard approach includes several weak points:

(1) The lab observer can perform transformation of the velocities of the two particles (the charged particle and the neutral one), to his system of reference. We recall that the velocities of the two particles as observed by the freely-falling observer are equal, and the transformations of the velocities are of pure kinematic nature. If the two velocities were equal in the inertial system, they will also be equal in the lab system of reference, although one of the particles is radiating in the lab system. The question now arises, what is the source of the energy carried by the radiation from the charged particle?

(2) Another question arises: is the radiation emitted by a source an objective phenomenon, or is it a relative phenomenon, that exists for one observer, and does not exist for another? This question may be put more strongly: Suppose that the lab observer that observes the radiation, finds that the radiation is absorbed by another object, and causes some physical process in that object, like a transition to an excited energy level. Would the freely falling observer observe this process? What will be his explanation for the energy source that created this physical process?

These questions reveal a contradiction in the standard approach. The existence of radiation from a certain source is a universal phenomenon that can be observed, directly or indirectly, 
by any observer, without regard to his state of motion. We shall show later that the relative acceleration considered by Rohrlich as the condition for the observation of radiation, is actually the relative acceleration between the accelerated charge and its own electric field, and not between the charge and the observer.

(3) Another problem concerned with uniform acceleration is the vanishing of the radiation reaction force (see also Singal $\left.{ }^{(5)} 1997\right)$.

The Dirac-Lorentz equation for a charged particle is:

$$
m a^{\mu}=F_{i n}^{\mu}+F_{e x t}^{\mu}+\Gamma^{\mu}
$$

where $m$ is the particle mass, $a^{\mu}$ is the 4 -vector acceleration, $F_{i n}^{\mu}$ is the incoming radiation field, $F_{e x t}^{\mu}$ is the external force that drives the particle, and $\Gamma^{\mu}$ is Abraham four-vector of the radiation reaction:

$$
\Gamma^{\mu}=\frac{2 e^{2}}{3 c^{3}}\left(\dot{a}^{\mu}-\frac{1}{c^{2}} a^{\lambda} a_{\lambda} v^{\mu}\right)
$$

where $v^{\mu}$ is the 4-vector velocity of the source: $v^{\mu}=\left(\begin{array}{c}\gamma c \\ \gamma \vec{v}\end{array}\right)$.

$\Gamma$ was considered the "radiation-reaction force", and the work done against this force, was considered as the source for the energy carried by the radiation. The first term in $\Gamma, \frac{2 e^{2}}{3 c^{3}} \dot{a}^{\mu}$, is called the "Schott" term. For a uniformly accelerated particle, the motion is a hyperbolic motion (Rindler $\left.{ }^{(6)} 1966\right), \dot{a}^{\mu}=\frac{1}{c^{2}} a^{\lambda} a_{\lambda} v^{\mu}$, and $\Gamma^{\mu}$ vanishes.

The problem of the vanishing of the radiation reaction force is called the "energy balance paradox", where the vanishing of this force in such a motion gives rise to the question, what is the source of the energy carried away by the radiation. Several solutions were suggested for this paradox. Let us mention here the one suggested by Leibovitz and Peres ${ }^{(7)}$ (1963). This solution assumes the existence of an infinite charged plane, whose total charge is equal and opposite in sign to the charge of the accelerated particle, and it recesses with the speed of light in the opposite direction to that of the acceleration. The interaction between this plane and the accelerated charge 
is the source of the energy carried by the radiation. This solution is far from being satisfactory.

The solution suggested by the standard approach (Rohrolich ${ }^{(1)} 1965$ ) assumes that the Schott term, which is a part of "Abraham four-vector" is somehow isolated from the radiation reaction force, and is adaptted to the acceleration term in the equation of motion. According to this approach, the radiation reaction force is only: $\frac{-2 e^{2}}{3 c^{3}} \frac{1}{c^{2}} a^{\lambda} a_{\lambda} v^{\mu}$. To solve the equation of motion in its new form, a multiplication by an integration factor $\left(e^{-\tau / \tau_{0}}\right)$ is needed. This term, that includes the proper time in an exponential form, leads later to divergent solutions that must be discarded. The inclusion of the Schott term with the acceleration brings up another difficulty: the acceleration is influenced by a force that will act a short time later. This "backward action" in time (preacceleration), violates causality. Rohrlich ${ }^{(1)}$ (1965) argues that this backward action takes place over time scale that cannot be measured experimentally. However, if the Schott term is left with the "force" term, it is cancelled out in the case of uniform motion, by the second term in $\Gamma^{\mu}$, and the above mentioned difficulties are avoided.

(4) Another difficulty concerned with the standard approach (see also Singal (5) 1997), is the fact that for a zero velocity, the radiation from the uniformly accelerated charge has a mirror symmetry with respect to a plane located at the charge, perpendicular to the direction of the acceleration (see Jackson ${ }^{(8)}$ 1975, eq. 14.39). Due to this symmetry, the radiation imparts no momentum to the radiating charge, and there is indeed, no reaction force of the radiation on the radiating charge. This consideration justifies the vanishing of $\Gamma^{\mu}$ (which represents the radiation reaction force), and the removal of the Schott term from this expression leaves us with a radiation reaction force which physically does not exist.

The solution to the energy balance paradox is found in an alternative approach, proposed in the present work. We find that the electric field of an accelerated charge is curved relative to the instantanous system of reference of the charge, and the interaction between the charge and its own curved electric field gives rise to the radiation. In this solution the assumption about the Schott term is not necessary and the difficulties that emerge from the use of this assumption are avoided, nor an assumption of an infinite charged plane is needed (Leibovitz and Peres $\left.{ }^{(7)} 1963\right)$. 
The source of the radiated energy is found in the interaction between the accelerated charge and its own curved electric field.

Recently, two new papers that deal with the question of accelerated charge appeared in the literature (Singal (5) 1997, Parrot (9) 1997). Singal, after criticizing the standard approach, concludes that a uniformly accelerated charge does not radiate. This conclusion resembles that of Rosen ${ }^{(10)}$ (1962), who used similar arguements to conclude that a freely falling charge does not radiate. We do not agree with the conclusion of Singal, but we agree with the majority of his criticizm of the standard approach. Singal's points (i), (ii), (iii) (page 1372), raise the question of the radiation reaction force, and indeed point at inconsistencies in the standard approach. We agree with this criticizm, and we believe that our conjencture about the interaction of the accelerated charge with its own curved electric field, supplies the correct answer to this problem. Singal's point (iv) is that the motion of a uniformly accelerated charge does not contain any intrinsic time scale, that can determine a characteristic frequency for the radiation. We claim that there is an intrinsic time scale, defined similarily to the definition given by Jackson ${ }^{(8)}$ (1974, pp 667). Following his approach, we define a characteristic frequency $\omega_{c}$ as: $\omega_{c}=c / R_{c} \sim a / c$, where $R_{c}$ is the radius of curvature of the electric field. This radius of curvature plays a crucial role in the creation of the stress force in the curved field (see eq. 4), which gives rise to the radiation. Parrot ${ }^{(9)}$ (1997) does not accept the conclusion of Singal and discusses this topic. Actually, he responds to an earlier paper of Singal ${ }^{(11)}$ (1995), and only partly to the present paper of Singal. He claimes that the fault in Singal's approach is in that he treats an "acceleration for all times". Parrot claims that such a treatment is not physical, and he also presents examples in which such a treatment leads to inconsistencies. He comes to the conclusion that a uniformly accelerated charge for an arbitrary long (but finite) time, does radiate. However, our approach is entirely different, and we do not lean on Parrot's arguements in our conjencture.

\section{The Problem}

According to classical electrodynamics the power radiated by an accelerated charged particle 
is $\left(\mathrm{Jackson}^{(8)} 1974\right)$

$$
P=\frac{2}{3} \frac{e^{2} a^{2}}{c^{3}}
$$

where $e$ is the particle charge, $a$ is its acceleration, and $c$ is the speed of light. The existence of a radiation depends on the existence of an acceleration. In the frame of special relativity, nonaccelerated charged particles do not radiate. Therefore, the existence of radiation provides an absolute measure to distinguish between accelerated charged particles and nonaccelerated ones (Rohrlich $^{(1)}$ 1965). The question is: when one speaks about acceleration, to what system this acceleration is related. In the standard approach, the acceleration is measured relative to the observer, and if the observer and the charge are accelerated with the same acceleration, their relative acceleration vanishes, and such an observer will not detect radiation, the same as an observer at rest when observing a static charge.

We argue that the calculations presented by Rohrlich $^{(1)}$ (1965) for this case are correct, but we present a different physical interpretation to these calculations. The relative acceleration that matters in the present case is the relative acceleration between the charge and its electric field.

Let us elaborate on this example in more detail: The electric field is defined as a property of space (around the electric charge). The field at each point $(\mathrm{x})$ tells us what force will act on a unit charge when located at this point. The field is induced on space by a charge. Once it is induced, it is an independent physical entity, and its behaviour is determined by the nature of space on which it is induced. When an electric charge is accelerated by an external (nongravitational) force, the space surrounding the charge is not affected by this force. The electric field created by the charge at each moment, is attributed to space, and its behaviour is determined by the space. Hence, the field is not accelerated by the external force, and the charge is accelerated relative to its field. This situation is the cause for the creation of the radiation field (Jackson ${ }^{(8)} 1974$, eq. 14.14)

Without stating it explicitly, Jackson considers an acceleration relative to an inertial frame of reference that can be describedd by special relativity. Ordinarily, when general relativity is considered, the inertial frame of reference should be replaced by a freely falling frame of reference 
characterized by a set of geodesics that cover this system. Acceleration now is related to the local system of geodesics. The electric field that was induced on space by the electric charge, follows the local sysmtem of geodesics, while the charged particle accelerates relative to this frame of reference. There exists a relative acceleration between the electric field that follows the system of reference defined by the the geodesics, and the accelerated charge that does not follow this system.

A freely falling charge in a uniform gravitational field, moves along a geodesic line, and it is not subjected to any external force. Any physical entity located in the same space is subjected to the same gravitational field, and will follow similar geodesics. The charge and the field created by this charge are located in the same frame of reference; and in that frame, their relative situation is similar to the one existing between a static charge and its field in a free space (Singal $\left.{ }^{(11)} 1995\right)$. We conclude, in accordance with Einstein principle of equivalence, that a freely-falling charge does not radiate.

This charge can be observed by an observer falling freely parallel to the charge. Certainly, this observer will not detect radiation. The standard approach came to the same conclusion (Rohrlich ${ }^{(1)}$ 1965). When a static observer, sitted in his lab at rest in the gravitational field, observes the same freely falling charge, the physical situation of the charge does not change. We conclude that this observer finds that the charge and its electric field have the same acceleration and no radiation can be emitted from the charge. The lab observer will come to the conclusion that a freely falling charge does not radiate. This is opposite to the conclusion of the standard approach.

We describe the electric field of a single charge as field lines emanating from the charge to infinity. Any change in the field, induced by the charge on the space surrounding it, proceeds with a finite velocity, $c$. The field lines of a charge, are related to the system of geodesics, and for a static charge in free space (where spherical symmetry holds), they are straight lines. The field lines are influenced by the situation of the charge at the moment they "leave" the charge. Once they left the charge, they are related to the local system of geodesics that characterizes the space, and are not influenced any more by the further motion of the charge. Hence, when a charge is 
accelerated in a free space, the local system of geodesics to which the field lines are related, forms a curved system relative to the instantaneous system of the charge. The lines of the electric field of the charge are curved lines in the system of the (accelerated) charge that created the field (see figure 2 in Singal (5) 1997). This curvature of the electric field gives rise to a stress force between the charge and its electric field, and the action of this force creates the radiation.

\section{The Energy Source of the Radiation}

Let us study quantitatively the work done by the stress force of the curved electric field. The radiation emitted by a charged particle moving at a constant acceleration, $a$, relative to the instantaneous rest frame of the charge has a cylindrical symmetry around the direction of acceleration. When we start with a zero velocity, there is a mirror symmetry with respect to a plane perpendicular to the acceleration. Therefore the radiation imparts no momentum to the radiating charge, and the force, $F_{\text {acc }}$, that creates the acceleration $a$ is the same as the force required to accelerate a neutral particle with the same mass and acceleration at zero velocity. The work done by this force creates the kinetic energy of the accelerated particle. For the creation of the radiation, an additional work is required. What force performs this work?

The force that creates the radiation is the force needed to overcome the stress force of the electric field of the particle, which is curved in the instantaneous rest frame of the particle. This extra force, has to be supplied by the external force, in addition to the force needed to create the kinetic energy of the accelerated object. Therefore the total work performed by the external force can be decomposed into two parts: one part creates the kinetic energy of the particle, and the second part creates the energy carried by the radiation. The energy balance paradox is thus solved, where the energy done in overcoming the stress force of the curved electric field, creates the energy carried by the radiation, although the radiation reaction force, $\Gamma^{\mu}$, vanishes for uniformly accelerated charge. We shall sum over the stress force of the field, $f_{E}$, and calculate the work done against this force.

In order to sum over $f_{E}$, we have to integrate over a sphere whose center is located on the 
charge. Naturally, such an integration involves a divergence (at the center). To avoid such a divergence, we take as the lower limit of the integration a small distance from the center, $r=c \Delta t$, (where $\Delta t$ is infinitsimal), and later we demand that $\Delta t \rightarrow 0$. We calculate the work done by the stress force in the volume defined by $c \Delta t<r<r_{u p}$, where $c^{2} / a \gg r_{u p} \gg c \Delta t$. These calculations are performed in a system of reference $S$, defined by the geodesics, and momentarily coincides with the frame of reference of the accelerated charge at the charge location, at time $t=0$.

The force per unit volume due to the electric stress is $f_{E}=E^{2} /\left(4 \pi R_{c}\right)$, where $E$ is the electric field, and $R_{c}$ is the radius of curvature of the field lines. The radius of curvature is calculated by using the formulae for hyperbolic motion (Rindler $\left.{ }^{(5)} 1966\right)$. It can be easily shown that in the limit of $a t \ll c$ the radius of curvature is $R_{c} \simeq c^{2} /(a \sin \theta)$, where $\theta$ is the angle between the initial direction of the field line and the acceleration (see figure 2 by Singal ${ }^{(5)}, 1997$ ). The force per unit volume due to the electric stress is

$$
f_{E}(r)=\frac{E^{2}(r)}{4 \pi R_{c}}=\frac{a \sin \theta}{c^{2}} \frac{e^{2}}{4 \pi r^{4}}
$$

where in the second equality we have substituted for the electric field $E=e / r^{2}$. The stress force is perpendicular to the direction of the field lines, so that the component of the stress force along the acceleration is $-f_{E}(r) \sin \phi$, where $\phi$ is the angle between the local field line and the acceleration. For very short intervals (where the direction of the field lines did not change much from their original direction) $\phi \sim \theta$, and we can write: $-f_{E}(r) \sin \phi \simeq-f_{E}(r) \sin \theta=\frac{-a \sin ^{2} \theta}{c^{2}} \frac{e^{2}}{4 \pi r^{4}}$. The dependence of this force on $\theta$ is similar to the dependence of the radiation distribution of an accelerated charge at zero velocity on $\theta$. Integration of this force over a spherical shell extending from $r=c \Delta t$ to $r_{\text {up }}$, where $c^{2} / a \gg r_{\text {up }} \gg c \Delta t$, yields the total force due to stress

$$
F_{E}(t)=2 \pi \int_{c \Delta t}^{r_{\mathrm{up}}} r^{2} d r \int_{0}^{\pi} \sin \theta d \theta\left[-f_{E}(r) \sin \theta\right]=-\frac{2}{3} \frac{a}{c^{2}} \frac{e^{2}}{c \Delta t}\left(1-\frac{c \Delta t}{r_{\mathrm{up}}}\right) .
$$

Clearly the second term in the parenthesis can be neglected. (Preliminary analysis done by us shows that, with certain limitations, nonconstant acceleration can be incorporated into our 
picture. This will be presented elsewhere.) The power supplied by the external force on acting against the electric stress is $P_{E}=-F_{E} v=-F_{E} a \Delta t$, where we substituted $v=a \Delta t$, and $v$ is the charge velocity in the system $S$, defined earlier, at time $t=\Delta t$. Substituting for $F_{E}$ we obtain

$$
P_{E}(t)=\frac{2}{3} \frac{a^{2} e^{2}}{c^{3}}
$$

This is the power radiated by the accelerated charged particle (eq. 3).

\section{Conclusions}

As stated earlier, the cause of the radiation of an accelerated charge is the electric stress created when the field lines of the charge are curved relative to the system of reference adjacent to the charge. The rate of work required to overcome the electric stress of the charged particle is equal to the power of the radiation emitted by the accelerated charge.

We already mentioned that for a zero velocity, the radiation of a charge accelerated at a uniform acceleration, $a$, imparts no momentum to the radiating charge. This is the cause why a radiation formed by a uniform acceleration does not create a radiation reaction force. Intuitively, one tends to consider the radiation reaction force as the force that contradicts the creation of radiation, and the work done through overcoming this force, is the work invested in creating the radiation. However, the fact that radiation formed by a uniform acceleration does not create a reaction force, raised difficulties about the energy balance of the system, and people tried to explain this difficulty in different ways. (e. g. Leibovitz \& Peres ${ }^{(7)} 1963$, Parrot ${ }^{(9)}$ 1997). The existence of the stress force of the curved electric field, supplies a simple explanation for the source of the energy of the radiation, which is the work done by the force that overcomes the stress force of the curved electric field. Thus the paradox of the energy balance in radiation of a uniformly accelerated charge is solved.

We would like to stress here again that the calculations carried by Rohrlich ${ }^{(1)}$ (1965) about the relative acceleration are correct, but we find that we should consider the relative acceleration 
between the charge and its (curved) electric field, rather than the relative acceleration between the charge and the observer.

Acknowledgment: During early stages of this project we benefited from our discussions with the late Nathan Rosen. We acknowledge useful discussions on this topic with Amos Ori, Hillel Livne, and Ofer Eyal.

We also acknowledge the comments of the referee that helped to improve the manuscript. 


\section{references}

(1) Rohrlich, F. 1965, in Classical Charged Particles, Addison-Wesley Pub. Co.

(2) Rohrlich, F. 1963, Annals of Physics, 22, 169.

(3) Coleman, S. 1961, Project Rand, RM- 2820-PR.

(4) Boulware, D. G. 1980, Annals of Physics, 124, 169.

(5) Singal, A. K. 1997, Gen. Rel. Grav., 29, 1371.

(6) Rindler, W. 1966, Special Relativity, Second Edition, Oliver and Boyd.

(7) Leibovitz, C. \& Peres, A. 1963, Annals of Physics, 25, 400.

(8) Jackson, J. D. 1975, Classical Electrodynamics, Second Edition, John Wiley \& Sons (New York).

(9) Parrot, S. 1997, Gen. Rel. Grav., 29, 1463.

(10) Rosen, N. 1962, Annals of Physics, 17, 269.

(11) Singal, A. K. 1995, Gen. Rel. Grav., 27, 953. 